\title{
Extremal Problems on the Generalized $(l, d)$-Equiangular System Points in the Case of Arbitrary Multidimensional Complex Spaces
}

\author{
Andrey Targonskii*, Irina Targonskaya \\ Zhytomyr Ivan Franko State University, Ukraine \\ targonsk@mail.ru, targonsk@zu.edu.ua
}

Keywords: module and argument in multidimensional complex space, inner radius of domain, logarithmic capacity, quadratic differential, piecewise-separating transformation, Green function, radial systems of points, variational formula.

Abstract. In the paper we construct a counterpart of classical results on the generalized $(l, d)$ - equiangular system points on the rays in the case of arbitrary multidimensional complex spaces.

\section{Introduction}

This paper belongs to the theory of extremal problems on classes of non-overlapping domain, in the case of arbitrary multidimensional complex spaces. The begin of these investigations associated, in space $\mathbb{C}$, with the paper of M.A. Lavrent'ev [1] in 1934. He found the maximum of some functional with respect to two simply connected domains with two fixed points. We note that this result was needed him for applying to some aerodynamics problems. In 1947, G.M. Goluzin solved a similar problem for three fixed points on the complex plane [2]. Then the topic began to evolve rapidly. In this connection we may recall papers of many authors, including Y.E. Alenitsina, M.A. Lebedev, J. Jenkins, P.M. Tamrazov, P.P. Kufareva and others. Using the idea of P.M. Tamrazov, in 1975 G.P. Bakhtin solved first the problem with so-called "free poles" on the unit circle, see, e.g., [3].

An important step for the development of this topic was papers of V.N. Dubinin. He developed a new method of research that is method of piecewise-separating transformation. He also first solved numerous of extremal problems for an arbitrary but fixed multiconnected non-overlapping domains (see, e.g., [4], [5], [6]). Now this type of extremal problems is used for investigations in holomorphic dynamics.

In the last decade actively used Bakhtin's method of "managing functional". He managed to solve a series of extremal problems for so-called "radial systems of points" (see, e.g., [4], [7]-[13]).

In the space $\mathbb{C}^{n}, n \geq 2$, the first results were obtained in [14]-[16]. In the present paper, we use these results.

\section{Theory in $\mathbb{C}$}

Let $\mathbb{N}, \mathbb{R}, \mathbb{C}$ be sets natural, real and complex numbers, conformably, $\overline{\mathbb{C}}=\mathbb{C} \bigcup\{\infty\}$ and $\mathbb{R}_{+}=(0, \infty)$. Then let $l, m, d \in \mathbb{N}$, thus $m=l d$. We will consider the set of natural numbers $\left\{m_{k}\right\}_{k=1}^{l}$ such that

$$
\sum_{k=1}^{l} m_{k}=m .
$$

System points

$$
A_{l, d}:=\left\{a_{k, p} \in \mathbb{C}: k=\overline{1, l}, p=\overline{1, m_{k}}\right\},
$$

we will called on the generalized $(l, d)$-equiangular system points on the rays, if she meets a condition (1), and at all $k=\overline{1, l}, p=\overline{1, m_{k}}$ the relations are executed:

$$
\begin{aligned}
& 0<\left|a_{k, 1}\right|<\ldots<\left|a_{k, m_{k}}\right|<\infty \\
& \arg a_{k, 1}=\arg a_{k, 2}=\ldots=\arg a_{k, m_{k}}=\frac{2 \pi}{l}(k-1) .
\end{aligned}
$$


Arbitrary generalized $(l, d)$-equiangular system points with the variable amount of points on the rays $A_{l, d}$ the set of domains is comparable $\left\{P_{k}\right\}_{k=1}^{l}$, where

$$
P_{k}:=\left\{w \in \mathbb{C} \backslash\{0\}: \frac{2 \pi}{l}(k-1)<\arg w<\frac{2 \pi}{l} k\right\}, k=\overline{1, l} .
$$

"Managing" functional we will consider for arbitrary generalized $(l, d)$-equiangular system points with the variable amount of points on the rays $A_{l, d}$

$$
\mu\left(A_{l, d}\right):=\prod_{k=1}^{l} \prod_{p=1}^{m_{k}}\left[\chi\left(\left|a_{k, p}\right|^{\frac{n}{2}}\right)\left|a_{k, p}\right|\right],
$$

where $\chi(t)=\frac{1}{2} \cdot\left(t+t^{-1}\right)$.

Let $\left\{B_{0}, B_{k, p}, B_{\infty}\right\}$ - arbitrary non-overlapping domains such, that

$$
0 \in B_{0}, a_{k, p} \in B_{k, p}, \infty \in B_{\infty}, B_{0}, B_{k, p}, B_{\infty} \subset \overline{\mathbb{C}}, \quad k=\overline{1, l}, p=\overline{1, m_{k}} .
$$

Let

$$
g_{B}(z, a)=h_{B, a}(z)+\log \frac{1}{|z-a|}
$$

generalized Green's function of domains $B$ with respect to a point $a \in B$. If $a=\infty$, then

$$
g_{B}(z, \infty)=h_{B, \infty}(z)+\log \frac{1}{|z|} .
$$

The value of

$$
r(B, a):=\exp \left(h_{B, a}(z)\right)
$$

the define of inner radius domain $B \subset \overline{\mathbb{C}}$ with respect to a point $a \in B$ (see [4], [5], [6], [17], [18], [19]).

We use the concept of a quadratic differential. Recall that a quadratic differential on a Riemann surface $S$ is a map

$$
\varphi: T S \rightarrow \mathbb{C}
$$

satisfying

$$
\varphi(\lambda v)=\lambda^{2} \varphi(v)
$$

for all $v \in T S$ and all $\lambda \in \mathbb{C}, T S$ - tangent space. If $z \in U \rightarrow \mathbb{C}$, is a chart defined on some open set $U \subset S$ then $\varphi$ is equal on $U$ to

$$
\varphi_{U}(z) d z^{2}
$$

for some function $\varphi_{U}$ defined on $z(U)$.

Suppose that two charts $z: U \rightarrow \mathbb{C}$ and $w: V \rightarrow \mathbb{C}$ on $S$ overlap, and let

$$
h:=w \circ z^{-1}
$$

be the transition function. If $\varphi$ is represented both as $\varphi_{U}(z) d z^{2}$ and $\varphi_{V}(w) d w^{2}$ on $U \cap V$, then we have

$$
\varphi_{V}(h(z))\left(h^{\prime}(z)\right)^{2}=\varphi_{U}(z) .
$$

One way to say this is that quadratic differentials transform under pull-backs by the square of the derivative. As the main results associated with it can be found in [20]. 
For arbitrary $l, d \in \mathbb{N}, n \geq 2$ let $A_{l, d}^{(1)}$ the define generalized $(l, d)$-equiangular system points the formed by poles of the quadratic differential $Q_{1}(w) d w^{2}$, where

$$
Q_{1}(w) d w^{2}=-\frac{w^{l-2}\left(1+w^{l}\right)^{2 d-1}}{\left[\left(1-i w^{\frac{l}{2}}\right)^{2 d+1}-\left(1+i w^{\frac{l}{2}}\right)^{2 d+1}\right]^{2}} d w^{2} .
$$

Let $A_{l, d}^{(2)}$ - the define generalized $(l, d)$-equiangular system points the formed by poles of the quadratic differential $Q_{2}(w) d w^{2}$, where

$$
Q_{2}(w) d w^{2}=\frac{w^{l-2}\left(1+w^{n}\right)^{2 d-1}}{\left[\left(1-i w^{\frac{l}{2}}\right)^{2 d+1}+\left(1+i w^{\frac{l}{2}}\right)^{2 d+1}\right]^{2}} d w^{2} .
$$

Let $A_{l, d}^{(3)}$ - the define generalized $(l, d)$-equiangular system points the formed by poles of the quadratic differential $Q_{3}(w) d w^{2}$, where

$$
Q_{3}(w) d w^{2}=-\frac{w^{l-2}\left(1+w^{l}\right)^{2 d}}{\left[\left(1-i w^{\frac{l}{2}}\right)^{2 d+2}-\left(1+i w^{\frac{l}{2}}\right)^{2 d+2}\right]^{2}} d w^{2}
$$

Let $A_{l, d}^{(4)}$ - the define generalized $(l, d)$-equiangular system points the formed by poles of the quadratic differential $Q_{4}(w) d w^{2}$, where

$$
Q_{4}(w) d w^{2}=-\frac{w^{l-2}\left(1+w^{l}\right)^{2 d-2}}{\left[\left(1-i w^{\frac{l}{2}}\right)^{2 d}+\left(1+i w^{\frac{l}{2}}\right)^{2 d}\right]^{2}} .
$$

For system points $A_{l, d}^{(1)}, A_{l, d}^{(2)}, A_{l, d}^{(3)}, A_{l, d}^{(4)}$ in ratios (2) the condition is satisfied $m_{k}=d, k=\overline{1, l}$.

\section{Theory in $\mathbb{C}^{n}$}

The main results of this section can be found in [14] - [16].

Space $\mathbb{C}^{n}$. It is a well-known fact that the complex space $\mathbb{C}^{n}$ is a linear vector space over the complex numbers with the Hermitian product

$$
(\mathbb{Z} \cdot \mathbb{W})=\sum_{k=1}^{n} z_{k} \bar{w}_{k}
$$

where $\mathbb{Z}=\left\{z_{k}\right\}_{k=1}^{n} \in \mathbb{C}^{n}, \mathbb{W}=\left\{w_{k}\right\}_{k=1}^{n} \in \mathbb{C}^{n}$.

Algebra $\mathbb{C}^{n}$. A binary operation acting from $\mathbb{C}^{n} \times \mathbb{C}^{n}$ into $\mathbb{C}^{n}$ by the rule

$$
\mathbb{Z} \cdot \mathbb{W}=\left\{z_{k} w_{k}\right\}_{k=1}^{n}
$$

is called vector multiplication of elements $\mathbb{C}^{n}$; here $\mathbb{Z}=\left\{z_{k}\right\}_{k=1}^{n} \in \mathbb{C}^{n}, \mathbb{W}=\left\{w_{k}\right\}_{k=1}^{n} \in \mathbb{C}^{n}$.

This operation turns $\mathbb{C}^{n}$ into a commutative, associative algebra with unit $\mathbf{1}=(1,1, \ldots, 1) \in \mathbb{C}^{n}$.

Elements $\mathbb{Z}=\left\{z_{k}\right\}_{k=1}^{n} \in \mathbb{C}^{n}$ such that $z_{k} \neq 0$ for all $k=\overline{1, n}$ are invertible with respect to the defined operation of multiplication. The inverse to such elements $\mathbb{Z} \in \mathbb{C}^{n}$ are the elements $\mathbb{Z}^{-1}=\left\{z_{k}^{-1}\right\}_{k=1}^{n} \in \mathbb{C}^{n}$, since $\mathbb{Z} \cdot \mathbb{Z}^{-1}=\mathbb{Z}^{-1} \cdot \mathbb{Z}=\mathbf{1}$.

We denote by $\Theta$ the set of all non-invertible elements, i.e. $a=\left\{a_{k}\right\}_{k=1}^{n} \in \mathbb{C}^{n}$ whose at least one coordinate $a_{k}=0$. For $n=1$ the equality (3) provides the usual multiplication of complex numbers. 
It is well-known that multiplication (3) admits a presentation of $\mathbb{C}^{n}$ as a direct sum of $n$ copies of the algebra $\mathbb{C}$.

Conjugation. One of the important notions in the algebra $\mathbb{C}$ is the notion of conjugate number.

Given $\mathbb{W}=\left\{w_{k}\right\}_{k=1}^{n} \in \mathbb{C}^{n}$ we introduce the vector conjugate element by $\overline{\mathbb{W}}=\left\{\bar{w}_{k}\right\}_{k=1}^{n} \in \mathbb{C}^{n}$, where $\bar{w}_{k}$ denotes the complex conjugate number $w_{k}$ in the usual sense.

The so defined correspondence is an automorphism $\mathbb{C}^{n}$ which leaves fixed the subspace $\mathbb{R}^{n} \subset \mathbb{C}^{n}$. For $n=1$ the vector conjugate number coincides with the complex conjugate one.

Vector module. An another crucial notion in the algebra $\mathbb{C}$ is the module of complex number. The following definition presents its analogue in $\mathbb{C}^{n}$.

Let $\mathbb{R}_{+}^{n}=\mathbb{R}_{+} \times \mathbb{R}_{+} \times \ldots \times \mathbb{R}_{+}$. A vector $|\mathbb{Z}|:=\left\{\left|z_{k}\right|\right\}_{k=1}^{n} \in \mathbb{R}_{+}^{n}$ is called the vector module of $\mathbb{Z}=\left\{z_{k}\right\}_{k=1}^{n} \in \mathbb{C}^{n}$.

Thus, the vector module is a mapping from $\mathbb{C}^{n}$ into $\mathbb{R}_{+}^{n}$. This embedding allows to obtain, in particular, the Reinhart domains in $\mathbb{C}^{n}$.

Note that for any $\mathbb{Z}=\left\{z_{k}\right\}_{k=1}^{n} \in \mathbb{C}^{n}$, we have

$$
\mathbb{Z} \cdot \overline{\mathbb{Z}}=|\overline{\mathbb{Z}}|^{2}=|\mathbb{Z}|^{2}
$$

Vector norm. A vector $\mathbb{X}=\left\{x_{k}\right\}_{k=1}^{n} \in \mathbb{R}^{n}$ is called non-negative and denoted by $\mathbb{X} \geq \mathbb{O}$, if $x_{k} \geq 0$ for all $k=\overline{1, n}$; here $\mathbb{O}=(0,0, \ldots, 0)$. If in addition $x_{k}>0$ for some $k$, we call $\mathbb{X}$ strongly positive.

We say that the vector $\mathbb{X}=\left\{x_{k}\right\}_{k=1}^{n} \in \mathbb{R}^{n}$ is greater than or equal to a vector $\mathbb{Y}=\left\{y_{k}\right\}_{k=1}^{n} \in \mathbb{R}^{n}$, if $\mathbb{X}-\mathbb{Y} \geq \mathbb{O}$, or correspondingly strongly greater when $\mathbb{X}-\mathbb{Y}>\mathbb{O}$.

These definitions for $n=1$ coincide with the usual comparison of real numbers.

For $n>1$ the situation becomes quite different. For example, the vector $\mathbb{O}$ is greater than or equal to all vectors whose coordinates are all non-positive and is less than or equal to all vectors from $\mathbb{R}_{+}^{n}$.

Vectors $\mathbb{X}$ with two coordinates of different signs are not comparable with the vector $\mathbb{O}$.

Now let us introduce a vector norm $\|\mathbb{Y}\|$ for any $\mathbb{Y} \in \mathbb{C}^{n}$ by

1) $\|\mathbb{Y}\| \geq \mathbb{O}$, and moreover $\|Y\|=\mathbb{O} \Longleftrightarrow \mathbb{Y}=\mathbb{O}$;

2) $\|\gamma \mathbb{Y}\|=|\gamma|\|\mathbb{Y}\| \quad \forall \gamma \in \mathbb{C}$

3) $\left\|\mathbb{Y}_{1}+\mathbb{Y}_{2}\right\| \leq\left\|\mathbb{Y}_{1}\right\|+\left\|\mathbb{Y}_{2}\right\| \quad \forall \mathbb{Y}_{1}, \mathbb{Y}_{2} \in \mathbb{C}^{n}$.

Thus, the vector module is a vector norm in the algebra $\mathbb{C}^{n}$. In this way an open unit ball in the algebra $\mathbb{C}^{n}$ is the open unit polydisk $\|\mathbb{Z}\|<\mathbf{1},(\mathbf{1}=(1,1, \ldots, 1))$, and a unit sphere is the $n$ dimensional torus $\mathbb{T}^{n}=\left\{\mathbb{Z} \in \mathbb{C}^{n}:\|\mathbb{Z}\|=1\right\}$.

Note that

a) $\left|\mathbb{Z}_{1} \cdot \mathbb{Z}_{2}\right|=|| \mathbb{Z}_{1} \cdot \mathbb{Z}_{2}||=\left\|\mathbb{Z}_{1}|||| \mathbb{Z}_{2}\right\|=\left|\mathbb{Z}_{1}\right|\left|\mathbb{Z}_{2}\right|, \forall \mathbb{Z}_{1}, \mathbb{Z}_{2} \in \mathbb{C}^{n}$

b) $|\mathbf{1}|=\|\mathbf{1}\|=\mathbf{1}$.

Vector argument. Now we define a vector argument of $n$-dimensional complex numbers $\mathbb{A}=$ $\left\{a_{k}\right\}_{k=1}^{n} \in \mathbb{C}^{n} \backslash \Theta$ as an $n$-dimensional real vector by

$$
\operatorname{Arg} \mathbb{A}=\left\{\operatorname{Arg} a_{k}\right\}_{k=1}^{n}
$$

Representation of $n$-dimensional complex number in vector cartesian form. For any $\mathbb{Z}=$ $\left\{z_{k}\right\}_{k=1}^{n} \in \mathbb{C}^{n}$ one can write

$$
\begin{gathered}
\mathbb{Z}=\left\{z_{k}\right\}_{k=1}^{n}=\left\{\operatorname{Re} z_{k}+i \operatorname{Im} z_{k}\right\}_{k=1}^{n}=\left\{\operatorname{Re} z_{k}\right\}_{k=1}^{n}+\left\{i \operatorname{Im} z_{k}\right\}_{k=1}^{n}= \\
=\left\{\operatorname{Re} z_{k}\right\}_{k=1}^{n}+i\left\{\operatorname{Im} z_{k}\right\}_{k=1}^{n}=\operatorname{Re} \mathbb{Z}+i \operatorname{Im} \mathbb{Z}=\mathbb{X}+i \mathbb{Y}= \\
=\left\{x_{k}\right\}_{k=1}^{n}+i\left\{y_{k}\right\}_{k=1}^{n} \in \mathbb{R}^{n}+i \mathbb{R}^{n},
\end{gathered}
$$


where $\mathbb{X}=\operatorname{Re} \mathbb{Z}=\left\{\operatorname{Re} z_{k}\right\}_{k=1}^{n}=\left\{x_{k}\right\}_{k=1}^{n}, \mathbb{Y}=\operatorname{Im} \mathbb{Z}=\left\{\operatorname{Im} z_{k}\right\}_{k=1}^{n}=\left\{y_{k}\right\}_{k=1}^{n}$. Thus, $\mathbb{C}^{n}=$ $\mathbb{R}^{n}+i \mathbb{R}^{n}$.

Presentation of $n$-dimensional complex numbers in vector polar form. We can write the elements $\mathbb{Z}$ of $\mathbb{C}^{n}$ in the form

$$
\begin{gathered}
\mathbb{Z}=\left(\begin{array}{c}
z_{1} \\
z_{2} \\
\vdots \\
z_{n}
\end{array}\right)=\left(\begin{array}{c}
\left|z_{1}\right| e^{i \alpha_{1}} \\
\left|z_{2}\right| e^{i \alpha_{2}} \\
\vdots \\
\left|z_{n}\right| e^{i \alpha_{n}}
\end{array}\right)=\left(\begin{array}{c}
\left|z_{1}\right| \\
\left|z_{2}\right| \\
\vdots \\
\left|z_{n}\right|
\end{array}\right)\left(\begin{array}{c}
e^{i \alpha_{1}} \\
e^{i \alpha_{2}} \\
\vdots \\
e^{i \alpha_{n}}
\end{array}\right)= \\
=|\mathbb{Z}|\left[\left(\begin{array}{c}
\cos \alpha_{1} \\
\cos \alpha_{2} \\
\vdots \\
\cos \alpha_{n}
\end{array}\right)+i\left(\begin{array}{c}
\sin \alpha_{1} \\
\sin \alpha_{2} \\
\vdots \\
\sin \alpha_{n}
\end{array}\right)\right]=|\mathbb{Z}|[\cos \operatorname{Arg} \mathbb{Z}+i \sin \operatorname{Arg} \mathbb{Z}]= \\
=|\mathbb{Z}| e^{i \operatorname{Arg} \mathbb{Z}}=|\mathbb{Z}| \exp i \operatorname{Arg} \mathbb{Z},
\end{gathered}
$$

where

$$
\begin{gathered}
\cos \beta=\left(\begin{array}{c}
\cos \beta_{1} \\
\cos \beta_{2} \\
\vdots \\
\cos \beta_{n}
\end{array}\right), \quad \sin \beta=\left(\begin{array}{c}
\sin \beta_{1} \\
\sin \beta_{2} \\
\vdots \\
\sin \beta_{n}
\end{array}\right), \\
\exp i \beta=\left(\begin{array}{c}
\exp i \beta_{1} \\
\exp i \beta_{2} \\
\vdots \\
\exp i \beta_{n}
\end{array}\right), \quad \beta=\left(\begin{array}{c}
\beta_{1} \\
\beta_{2} \\
\vdots \\
\beta_{n}
\end{array}\right) \in \mathbb{R}^{n}, \quad \mathbb{Z}=\left(\begin{array}{c}
z_{1} \\
z_{2} \\
\vdots \\
z_{n}
\end{array}\right) \in \mathbb{C}^{n} .
\end{gathered}
$$

A polycylindrical domain in $\overline{\mathbb{C}^{n}}$ is defined a follows:

$$
\mathbb{B}=B_{1} \times B_{2} \times \ldots \times B_{n}, \quad B_{q} \subset \overline{\mathbb{C}}, q=1,2, . ., n .
$$

The domains $B_{q}, q=1,2, . ., n$, are called coordinate domains.

Let $\Omega\left(a_{1}, a_{2}, \ldots, a_{n}\right)$ be the point of the space $\mathbb{C}^{n}$ with coordinates $a_{q}, q=1,2, \ldots, n$.

A generalized inner radius of the polycylindrical domain $\mathbb{B}$ at the point $\Omega, \Omega \in \mathbb{B}$ is defined as follows:

$$
\mathbf{r}(\mathbb{B}, \Omega)=\left(\prod_{q=1}^{n} r\left(B_{q}, a_{q}\right)\right)^{\frac{1}{n}}
$$

where $r\left(B_{q}, a_{q}\right)$ is the inner radius of the coordinate domain $B_{q}$ at the point $a_{q}$.

\section{Some results in the theory non-overlapping domains}

In this section we mention some of the results in the space $\mathbb{C}$, which will be carried by us in the space $\mathbb{C}^{n}$.

Theorem 1 [11]. Let $l, m, d \in \mathbb{N}, m=l d, l \geq 2$. Then for arbitrary generalized $(l, d)$-equiangular system points $A_{l, d}=\left\{a_{k, p}\right\}$ the satisfied condition (2),

$$
\mu\left(A_{l, d}\right)=\mu\left(A_{l, d}^{(1)}\right)
$$


with set of numbers $\left\{m_{k}\right\}_{k=1}^{l}$ the satisfied condition (1), and arbitrary set non-overlapping domains $\left\{B_{0}, B_{k, p}\right\}, 0 \in B_{0}, a_{k, p} \in B_{k, p}, B_{0}, B_{k, p} \subset \overline{\mathbb{C}}$, be satisfied inequality

$$
r^{\frac{l^{2}}{4}}\left(B_{0}, 0\right) \cdot \prod_{k=1}^{l} \prod_{p=1}^{m_{k}} r\left(B_{k, p}, a_{k, p}\right) \leq\left(\frac{8}{2 m+l}\right)^{m} \cdot\left(\frac{2 l}{2 m+l}\right)^{\frac{l}{2}} \cdot \mu\left(A_{l, d}^{(1)}\right) .
$$

Theorem 2. [11]. Let $l, m, d \in \mathbb{N}, m=l d, l \geq 2$. Then for arbitrary generalized $(l, d)$-equiangular system points $A_{l, d}=\left\{a_{k, p}\right\}$ the satisfied condition (2),

$$
\mu\left(A_{l, d}\right)=\mu\left(A_{l, d}^{(2)}\right)
$$

with set of numbers $\left\{m_{k}\right\}_{k=1}^{l}$ the satisfied condition (1), and arbitrary set non-overlapping domains $\left\{B_{k, p}, B_{\infty}\right\}, \infty \in B_{\infty}, a_{k, p} \in B_{k, p}, B_{k, p}, B_{\infty} \subset \overline{\mathbb{C}}$, be satisfied inequality

$$
r^{\frac{l^{2}}{4}}\left(B_{\infty}, \infty\right) \cdot \prod_{k=1}^{l} \prod_{p=1}^{m_{k}} r\left(B_{k, p}, a_{k, p}\right) \leq\left(\frac{8}{2 m+l}\right)^{m} \cdot\left(\frac{2 l}{2 m+l}\right)^{\frac{l}{2}} \cdot \mu\left(A_{l, d}^{(2)}\right) .
$$

Theorem 3. [9]. Let $l, m, d \in \mathbb{N}, m=l d, l \geq 2$. Then for arbitrary generalized $(l, d)$-equiangular system points $A_{l, d}=\left\{a_{k, p}\right\}$ the satisfied condition (2),

$$
\mu\left(A_{l, d}\right)=\mu\left(A_{l, d}^{(3)}\right)
$$

with set of numbers $\left\{m_{k}\right\}_{k=1}^{l}$ the satisfied condition (1), and arbitrary set non-overlapping domains $\left\{B_{0}, B_{k, p}, B_{\infty}\right\}, 0 \in B_{0}, \infty \in B_{\infty}, a_{k, p} \in B_{k, p}, B_{0}, B_{k, p}, B_{\infty} \subset \overline{\mathbb{C}}$, be satisfied inequality

$$
\left(r\left(B_{0}, 0\right) \cdot r\left(B_{\infty}, \infty\right)\right)^{\frac{l^{2}}{4}} \cdot \prod_{k=1}^{l} \prod_{p=1}^{m_{k}} r\left(B_{k, p}, a_{k, p}\right) \leq\left(\frac{4}{l+m}\right)^{m} \cdot\left(\frac{l}{l+m}\right)^{l} \cdot \mu\left(A_{l, d}^{(3)}\right) .
$$

Theorem 4. [10]. Let $l, m, d \in \mathbb{N}, m=l d, l \geq 2$. Then for arbitrary generalized $(l, d)$-equiangular system points $A_{l, d}=\left\{a_{k, p}\right\}$ the satisfied condition (2),

$$
\mu\left(A_{l, d}\right)=\mu\left(A_{l, d}^{(4)}\right)
$$

with set of numbers $\left\{m_{k}\right\}_{k=1}^{l}$ the satisfied condition (1), and arbitrary set non-overlapping domains $\left\{B_{k, p}\right\}, a_{k, p} \in B_{k, p} \subset \overline{\mathbb{C}}$ be satisfied inequality

$$
\prod_{k=1}^{l} \prod_{p=1}^{m_{k}} r\left(B_{k, p}, a_{k, p}\right) \leq\left(\frac{4}{l d}\right)^{l d} \cdot \mu\left(A_{l, d}^{(4)}\right)
$$

Main results in the space $\mathbb{C}^{n}$

Theorem 5. Let $l, m, d \in \mathbb{N}, m=l d, l \geq 2$, set of numbers $\left\{m_{k}\right\}_{k=1}^{l}$ the satisfied condition (1). Consider a system polycylindrical domains

$$
\mathbb{B}_{0}=B_{1}^{(0)} \times B_{2}^{(0)} \times \ldots \times B_{n}^{(0)}
$$




$$
\mathbb{B}_{k, p}=B_{1}^{(k, p)} \times B_{2}^{(k, p)} \times \ldots \times B_{n}^{(k, p)}, \quad B_{q}^{(0)}, B_{q}^{(k, p)} \subset \overline{\mathbb{C}}, q=\overline{1, n}, k=\overline{1, l}, p=\overline{1, m_{k}},
$$

and points

$$
\Omega_{0}=(0,0, \ldots, 0), \Omega_{k, p}=\left(a_{1}^{(k, p)}, a_{2}^{(k, p)}, \ldots, a_{n}^{(k, p)}\right), k=\overline{1, l}, p=\overline{1, m_{k}},
$$

in the space $\mathbb{C}^{n}$ satisfying the following conditions:

1) $\Omega_{0} \in \mathbb{B}_{0}, \Omega_{k, p} \in \mathbb{B}_{k, p}, k=\overline{1, l}, p=\overline{1, m_{k}}$,

2) For $q=1,2, \ldots, n$ system points $\left\{a_{q}^{(k, p)}, a_{q}^{(k, p)}, \ldots, a_{q}^{(k, p)}\right\}, n \geq 2$, is the generalized $(l, d)$ equiangular system points on the rays in space $\mathbb{C}$, the satisfied condition (2),

$$
\mu\left(A_{l, d}\right)=\mu\left(A_{l, d}^{(1)}\right)
$$

$k=\overline{1, l}, p=\overline{1, m_{k}}$.

3) For $q=1,2, . ., n$, the domains $B_{q}^{(0)}, B_{q}^{(k, p)}, B_{q}^{(k, p)}, \ldots, B_{q}^{(k, p)}, n \geq 2$, are pairwise disjoint in $\overline{\mathbb{C}}$, $k=\overline{1, l}, p=\overline{1, m_{k}}$.

Then the following inequality is true:

$$
\mathbf{r}^{\frac{l^{2}}{4}}\left(\mathbb{B}_{0}, \Omega_{0}\right) \cdot \prod_{k=1}^{l} \prod_{p=1}^{m_{k}} \mathbf{r}\left(\mathbb{B}_{k, p}, \Omega_{k, p}\right) \leq\left(\frac{8}{2 m+n}\right)^{m} \cdot\left(\frac{2 l}{2 m+n}\right)^{\frac{n}{2}} \cdot \mu\left(A_{n, d}^{(1)}\right) .
$$

Theorem 6. Let $l, m, d \in \mathbb{N}, m=l d, l \geq 2$, set of numbers $\left\{m_{k}\right\}_{k=1}^{l}$ the satisfied condition (1). Consider a system polycylindrical domains

$$
\begin{gathered}
\mathbb{B}_{\infty}=B_{1}^{(\infty)} \times B_{2}^{(\infty)} \times \ldots \times B_{n}^{(\infty)} \\
\mathbb{B}_{k, p}=B_{1}^{(k, p)} \times B_{2}^{(k, p)} \times \ldots \times B_{n}^{(k, p)}, \quad B_{q}^{(\infty)}, B_{q}^{(k, p)} \subset \overline{\mathbb{C}}, q=\overline{1, n}, k=\overline{1, l}, p=\overline{1, m_{k}},
\end{gathered}
$$

and points

$$
\Omega_{\infty}=(\infty, \infty, \ldots, \infty), \Omega_{k, p}=\left(a_{1}^{(k, p)}, a_{2}^{(k, p)}, \ldots, a_{n}^{(k, p)}\right), k=\overline{1, l}, p=\overline{1, m_{k}},
$$

in the space $\mathbb{C}^{n}$ satisfying the following conditions:

1) $\Omega_{\infty} \in \mathbb{B}_{\infty}, \Omega_{k, p} \in \mathbb{B}_{k, p}, k=\overline{1, l}, p=\overline{1, m_{k}}$,

2) For $q=1,2, \ldots, n$ system points $\left\{a_{q}^{(k, p)}, a_{q}^{(k, p)}, \ldots, a_{q}^{(k, p)}\right\}, n \geq 2$, is the generalized $(l, d)$ equiangular system points on the rays in space $\mathbb{C}$, the satisfied condition (2),

$$
\mu\left(A_{l, d}\right)=\mu\left(A_{l, d}^{(2)}\right)
$$

$k=\overline{1, l}, p=\overline{1, m_{k}}$.

3) For $q=1,2, . ., n$, the domains $B_{q}^{(k, p)}, B_{q}^{(k, p)}, \ldots, B_{q}^{(k, p)}, B_{q}^{(\infty)}, n \geq 2$, are pairwise disjoint in $\overline{\mathbb{C}}, k=\overline{1, l}, p=\overline{1, m_{k}}$.

Then the following inequality is true:

$$
\mathbf{r}^{\frac{l^{2}}{4}}\left(\mathbb{B}_{\infty}, \Omega_{\infty}\right) \cdot \prod_{k=1}^{l} \prod_{p=1}^{m_{k}} \mathbf{r}\left(\mathbb{B}_{k, p}, \Omega_{k, p}\right) \leq\left(\frac{8}{2 m+l}\right)^{m} \cdot\left(\frac{2 l}{2 m+l}\right)^{\frac{l}{2}} \cdot \mu\left(A_{l, d}^{(2)}\right) .
$$


Theorem 7. Let $l, m, d \in \mathbb{N}, m=l d, l \geq 2$, set of numbers $\left\{m_{k}\right\}_{k=1}^{l}$ the satisfied condition (1). Consider a system polycylindrical domains

$$
\begin{gathered}
\mathbb{B}_{0}=B_{1}^{(0)} \times B_{2}^{(0)} \times \ldots \times B_{n}^{(0)}, \mathbb{B}_{\infty}=B_{1}^{(\infty)} \times B_{2}^{(\infty)} \times \ldots \times B_{n}^{(\infty)}, \\
\mathbb{B}_{k, p}=B_{1}^{(k, p)} \times B_{2}^{(k, p)} \times \ldots \times B_{n}^{(k, p)}, \quad B_{q}^{(0)}, B_{q}^{(\infty)}, B_{q}^{(k, p)} \subset \overline{\mathbb{C}}, q=\overline{1, n}, k=\overline{1, l}, p=\overline{1, m_{k}},
\end{gathered}
$$

and points

$$
\Omega_{0}=(0,0, \ldots, 0), \Omega_{\infty}=(\infty, \infty, \ldots, \infty), \Omega_{k, p}=\left(a_{1}^{(k, p)}, a_{2}^{(k, p)}, \ldots, a_{n}^{(k, p)}\right), k=\overline{1, l}, p=\overline{1, m_{k}},
$$

in the space $\mathbb{C}^{n}$ satisfying the following conditions:

1). $\Omega_{0} \in \mathbb{B}_{0}, \Omega_{\infty} \in \mathbb{B}_{\infty}, \Omega_{k, p} \in \mathbb{B}_{k, p}, k=\overline{1, l}, p=\overline{1, m_{k}}$,

$2)$. For $q=1,2, \ldots, n$ system points $\left\{a_{q}^{(k, p)}, a_{q}^{(k, p)}, \ldots, a_{q}^{(k, p)}\right\}, n \geq 2$, is the generalized $(l, d)$ equiangular system points on the rays in space $\mathbb{C}$, the satisfied condition (2),

$$
\mu\left(A_{l, d}\right)=\mu\left(A_{l, d}^{(3)}\right)
$$

$k=\overline{1, l}, p=\overline{1, m_{k}}$.

3). For $q=1,2, . ., n$, the domains $B_{q}^{(0)}, B_{q}^{(k, p)}, B_{q}^{(k, p)}, \ldots, B_{q}^{(k, p)}, B_{q}^{(\infty)}, n \geq 2$, are pairwise disjoint in $\overline{\mathbb{C}}, k=\overline{1, l}, p=\overline{1, m_{k}}$.

Then the following inequality is true:

$$
\left(\mathbf{r}\left(\mathbb{B}_{0}, \Omega_{0}\right) \cdot \mathbf{r}\left(\mathbb{B}_{\infty}, \Omega_{\infty}\right)\right)^{\frac{l^{2}}{4}} \cdot \prod_{k=1}^{l} \prod_{p=1}^{m_{k}} \mathbf{r}\left(\mathbb{B}_{k, p}, \Omega_{k, p}\right) \leq\left(\frac{4}{l+m}\right)^{m} \cdot\left(\frac{l}{l+m}\right)^{l} \cdot \mu\left(A_{l, d}^{(3)}\right) .
$$

Theorem 8. Let $l, m, d \in \mathbb{N}, m=l d, l \geq 2$, set of numbers $\left\{m_{k}\right\}_{k=1}^{l}$ the satisfied condition (1). Consider a system polycylindrical domains

$$
\mathbb{B}_{k, p}=B_{1}^{(k, p)} \times B_{2}^{(k, p)} \times \ldots \times B_{n}^{(k, p)}, \quad B_{q}^{(k, p)} \subset \overline{\mathbb{C}}, q=\overline{1, n}, k=\overline{1, l}, p=\overline{1, m_{k}},
$$

and points

$$
\Omega_{k, p}=\left(a_{1}^{(k, p)}, a_{2}^{(k, p)}, \ldots, a_{n}^{(k, p)}\right), k=\overline{1, l}, p=\overline{1, m_{k}},
$$

in the space $\mathbb{C}^{n}$ satisfying the following conditions:

1) $\Omega_{k, p} \in \mathbb{B}_{k, p}, k=\overline{1, l}, p=\overline{1, m_{k}}$,

2) For $q=1,2, \ldots, n$ system points $\left\{a_{q}^{(k, p)}, a_{q}^{(k, p)}, \ldots, a_{q}^{(k, p)}\right\}, n \geq 2$, is the generalized $(l, d)$ equiangular system points on the rays in space $\mathbb{C}$, the satisfied condition (2),

$$
\mu\left(A_{l, d}\right)=\mu\left(A_{l, d}^{(4)}\right)
$$

$k=\overline{1, l}, p=\overline{1, m_{k}}$.

3) For $q=1,2, . ., n$, the domains $B_{q}^{(k, p)}, B_{q}^{(k, p)}, \ldots, B_{q}^{(k, p)}, n \geq 2$, are pairwise disjoint in $\overline{\mathbb{C}}$, $k=\overline{1, l}, p=\overline{1, m_{k}}$.

Then the following inequality is true:

$$
\prod_{k=1}^{l} \prod_{p=1}^{m_{k}} \mathbf{r}\left(\mathbb{B}_{k, p}, \Omega_{k, p}\right) \leq\left(\frac{4}{l d}\right)^{l d} \cdot \mu\left(A_{l, d}^{(4)}\right)
$$


Proof of Theorem 5. Using the definition of a space $\mathbb{C}^{n}$ inner radius, we have

$$
\mathbf{r}\left(\mathbb{B}_{0}, \Omega_{0}\right)=\left(\prod_{q=1}^{n} r\left(B_{q}^{(0)}, 0\right)\right)^{\frac{1}{n}}, \quad \mathbf{r}\left(\mathbb{B}_{k, p}, \Omega_{k, p}\right)=\left(\prod_{q=1}^{n} r\left(B_{q}^{(k, p)}, a^{(k, p)}\right)\right)^{\frac{1}{n}}
$$

where, respectively, $r\left(B_{q}^{(0)}, 0\right)$ and $r\left(B_{q}^{(k, p)}, a_{k, p}\right)$ - inner radius domains $B_{q}^{(0)}$ and $B_{q}^{(k, p)}$, with respect to a points $0 \in B_{q}^{(0)}$ and $a_{k, p} \in B_{q}^{(k, p)}, q=1,2, . ., n$ in space $\mathbb{C}$.

Then,

$$
\mathbf{r}^{\frac{l^{2}}{4}}\left(\mathbb{B}_{0}, \Omega_{0}\right) \cdot \prod_{k=1}^{l} \prod_{p=1}^{m_{k}} \mathbf{r}\left(\mathbb{B}_{k, p}, \Omega_{k, p}\right)=\prod_{q=1}^{n}\left(r^{\frac{l^{2}}{4}}\left(B_{q}^{(0)}, 0\right) \cdot \prod_{k=1}^{l} \prod_{p=1}^{m_{k}} r\left(B_{q}^{(k, p)}, a^{(k, p)}\right)\right)^{\frac{1}{n}} .
$$

Using Theorem 4, we obtain the final result. The theorem is proved.

Similarly, we prove Theorems 6, 7,8.

Acknowledgement. The author is grateful to Prof. A. Bakhtin for suggesting problems and useful discussions. This research is partially supported by Grant of Ministry of Education and Science of Ukraine (Project No. 0115U003027).

\section{References}

[1] M.A. Lavrent'ev, On the theory of conformal mappings, Tr. Fiz.-Mat. Inst. Akad. Nauk SSSR. 5 (1934) 159-245. (in Russian)

[2] G.M. Goluzin, Geometric theory of functions of a complex variable, Nauka, Moscow, 1966. (in Russian)

[3] G.P. Bakhtina, Variational methods and quadratic differentials in problems for disjoint domains, $\mathrm{PhD}$ thesis, Kiev, Ukrainian SSR, 1975. (in Russian)

[4] A.K. Bakhtin, G.P. Bakhtina, Yu.B. Zelinskii, Topological-algebraic structures and geometric methods in complex analysis, Inst. Math. NAS Ukraine, Kiev, Ukraine, 2008. (in Russian)

[5] V.N. Dubinin Separating transformation of domains and problems of extremal division, Zap. Nauchn. Sem. Leningrad. Otdel. Mat. Inst. Ros. Akad. Nauk. 168 (1988) 48-66. (in Russian)

[6] V.N. Dubinin, Method of symmetrization in the geometric theory of functions of a complex variable, Usp. Mat. Nauk. 49(1) (1994) 3-76.

[7] A.K. Bakhtin, Inequalities for the inner radii of nonoverlapping domains and open sets, Ukr. Math. J. 61(5) (2009) 716-733.

[8] A.K. Bakhtin, A.L. Targonskii, Extremal problems and quadratic differential, Nonlin. Oscillations. 8(3) (2005) 296-301.

[9] A.K. Bakhtin, A.L. Targonskii, Generalized (n, d)-ray systems of points and inequalities for nonoverlapping domains and open sets, Ukr. Math. J. 63(7) (2011) 999-1012.

[10] S.A. Ochrimenko, , A.L. Targonskii, Extreme problems for generalized ray systems of points, Zb. Praz. Ins-tu Matemat. NAN Ukrainy. 9(2) (2012) 270-284. (in Russian) 
[11] A. Targonskii, Extremal problems on the generalized (n; d)-equiangular system of points, An. St. Univ. Ovidius Constanta. 22(2) (2014) 239-251.

[12] A.L. Targonskii, Extremal problems for partially non-overlapping domains on equiangular systems of points, Bull. Soc. Sci. Lett. Lodz. 63(1) (2013) 57-63.

[13] A. Targonskii, I. Targonskaya, On the one extremal problem on the Riemann sphere, International Journal of Advanced Research in Mathematics. 4 (2016) 1-7.

[14] A.K. Bakhtin, Analytic functions of vector argument and partially-conformal mappings in multidimensional complex spaces, in: Progress in Analysis, Proceedings of the 8th Congress of the International Society for Analysis, its Applications, and Computation, 2011, pp. 1-8.

[15] A.K. Bakhtin, The generalization of some results of the theory of univalent functions on multidimensional complex spaces, Dop. NAN Ukr. 3 (2011) 7-11. (in Russian)

[16] Ya.V. Zabolotnyi, On one Dubinin extreme problem, Ukr. Math. J. 64(1) (2012) 24-34.

[17] V.N. Dubinin, Asymptotic representation of the modulus of a degenerating condenser and some its applications, Zap. Nauchn. Sem. Peterburg. Otdel. Mat. Inst. 237 (1997) 56-73. (in Russian)

[18] V.N. Dubinin, Capacities of condensers and symmetrization in geometric function theory of complex variables, Dal'nayka, Vladivostok, Russia, 2009. (in Russian)

[19] W.K. Hayman, Multivalent functions, Cambridge University, Cambridge, 1958.

[20] J.A. Jenkins, Univalent functions and conformal mapping, Springer, Berlin, 1958. 